\title{
Real-time Interactive Volumetric Animation of the Heart's Electrical Cycle from Automatically Synchronized ECG
}

\author{
John Ryan \\ Image Synthesis Group \\ Computer Science Dept. \\ Trinity College Dublin \\ Dublin 2, Ireland \\ john.t.ryan@cs.tcd.ie \\ http://isg.cs.tcd.ie
}

\author{
Carol O'Sullivan \\ Image Synthesis Group \\ Computer Science Dept. \\ Trinity College Dublin \\ Dublin 2, Ireland \\ osullica@tcd.ie \\ http://isg.cs.tcd.ie
}

\author{
Christopher \\ Bell \\ Physiology Dept. \\ Trinity College Dublin \\ Dublin 2, Ireland \\ cbell@tcd.ie
}

\begin{abstract}
We present a novel method for representing the electrical depolarisation and repolarisation of cardiac cells using real-time volumetric animation techniques. The visual representation coincides with automatically synchronized electrocardiogram (ECG) input. Whilst other projects in similar fields use super-computers or vastly complex cellular structures, one of the aims of this project is to simplify the process for real-time optimization. The current techniques are being used in a teaching tool and also are being implemented in a myocardial infarction diagnostic tool. Cellular automata are used to demonstrate the electrical progression with a multi-pass nearest-neighbour algorithm and automatic ECG recognition and detection algorithms are used to synchronize the animation with the inputted ECG. We use texture-based slices to represent the myocardial volume to improve speed.
\end{abstract}

Keywords: Virtual Reality, ECG simulation, cardiac modelling, texture-based volume

\section{Introduction}

Volumetric graphics programming techniques, along with ever-increasing computer specifications, are providing scope for rich representation of physiological phenomena. Slice-based volumization techniques enable real-time rendering of medical simulations and also enhance interactive capabilities. We present the animation system used in our teaching tool and current project of creating a diagnostic tool for the detection and monitoring of myocardial infarctions. This volumetric animation system runs in real-time with synchronized ECG input. Our heart model is a hybrid of slice-based volumetric and polygonal mesh graphics. Our ECG parameter measurement and detection software borrows well-established algorithms in ECG waveform analysis. The system is being developed for a diagnostic tool for myocardial infarctions (MIs). MI is the medical term for heart attack and is one of the leading causes of death throughout the world [2]. This paper builds on previous work [11] that had a crude animation system and lacked automation in the area of ECG synchronization. We have improved our earlier system by introducing a more accurate cellular structure, realistic electrical progressions, automatic ECG synchronization and speed optimisations.

The background to our research is reviewed in Section 2 with a short summary of the basic physiological/anatomical principles as well as graphical theory relevant to this system. In Section 3 we discuss how the data and models were attained as well as our model volumization and cellular representation techniques. In Section 4 we discuss our animation and synchronization techniques. Section 5 presents the results of our research and finally we conclude with a discussion of future work.

\section{Background}

Located centrally in the chest in a cavity called the mediastinum, the heart is responsible for pumping the blood around the 
body. It is generally made up of muscle and has a strong electrical presence. The adult heart weighs approximately $0.5 \mathrm{~kg}$ and it beats on average around 100,000 times a day. The heart has four chambers (two atria and two ventricles). The right atrium along with the venous system sucks deoxidised blood from most of the body and passes it to the right ventricle. The right ventricle then passes it to the lungs where oxidisation occurs and the blood is returned via the pulmonary vein to the left atrium. This blood is passed to the left ventricle, which outputs the regenerated blood to the body again via the aorta. (See Figure 1).

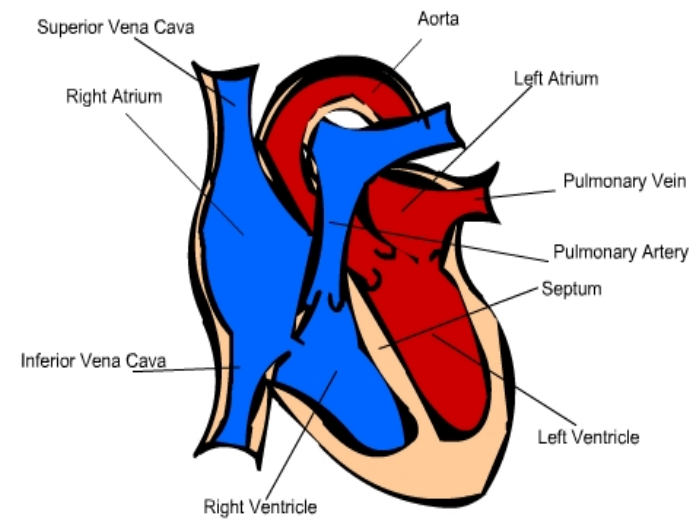

Figure 1. Diagram of the Heart

The recorded ECG displays a waveform in 2D. The voltage is represented vertically and time is represented horizontally. Sections of the wave coincide with physiological occurrences within the heart. Figure 1. shows an ECG that is currently used in our software.

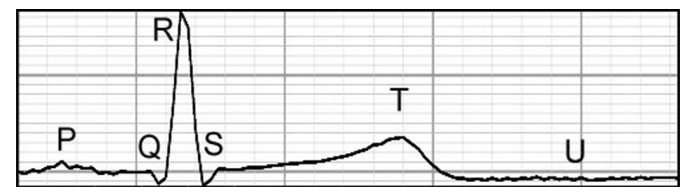

Figure 2. $E C G$

Figure 2 demonstrates a typical ECG from lead II in a standard three lead configuration. The different sections of the wave are also shown from $\mathrm{P}$ to $\mathrm{U}$. There are normally 12 leads in a standard clinical configuration. Within this configuration there are 3 standard leads, 3 augmented leads and 6 precordial leads. An electrical current travelling from the negative node to the positive node of a bipolar electrode is the basis of electrocardiography. This causes a positive deflection around the isoelectric line by the lead. The original three-lead bipolar electrode pair setup was first used by the inventor of ECG, Einthoven. It is still referred to as Einthoven's Triangle. (See Figure 3)

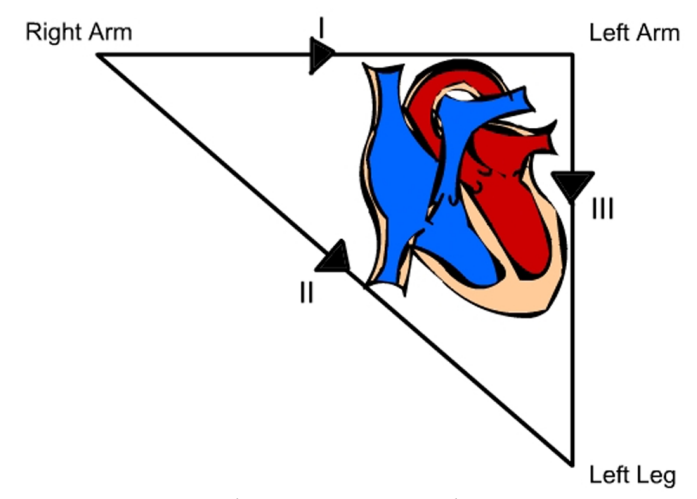

Figure 3. Einthoven's Triangle

Realistic models and simulations are becoming commonplace in medical education and practice due to the rapidly developing field of 3D graphics and virtual reality. This is aided by ever-increasing computer specifications and decreasing cost. VR training in medicine provides the student/practitioner with a greater sense of spatial awareness as well as not jeopardising a patient's safety [1]. Volume representation, 3D animation and interactivity are obvious advantages over $2 \mathrm{D}$ images. Interactivity is one of the most important features in a VR teaching tool. This however, becomes more difficult for the developer when working with volumetric data, but this process is becoming increasingly easy with techniques such as slice-based volume rendering [4]. In the field of cardiac simulation, the cellular datastructures are normally vast and require very long processing times or supercomputers [7]. CESLab [19] is a project based on commodity Apple Macintosh computers. That project is concerned with forward ECG simulation (i.e. Obtaining ECG from simulated cellular models), whereas we are generally interested in the inverse problem (i.e. Representing ECG data with graphical models). Although there is a myriad of papers such as that of Sermesant et al. [13] that deal with very detailed simulations of electrical 
propagation through the heart, we are more interested in simplified simulations suitable for real-time interaction.

Research has been conducted in the field of automatic ECG interpretation and pattern recognition for about 50 years [17]. However, it is still considered to be a very complex area of research. One main discipline in this field is that of pattern recognition and parameter measurement. With the results of these processes the ECG can be interpreted and any abnormalities can be detected. The first task of pattern recognition is unfortunately the most problematic. The QRS complex is a waveform that correlates with the excitation of the bundle-branches and ventricles. The most common application of this parameter is the measurement of heart rate. However, more importantly to our system, the other waveforms within the ECG can be found using this complex as a starting point. Köhler et al [6] found it difficult to select an "ideal" QRS detection algorithm since all were designed for different purposes. Algorithms were identified from many different fields including artificial neural networks [21], genetic algorithms [10], wavelet transforms [3], syntactic pattern recognition [17]. Tatara and Cinar [16] discuss a method of interpreting ECG data by integrating statistical and artificial intelligence tools. The So and Chan [14] QRS detection algorithm seems to be the most appropriate for our software because it was aimed at real-time systems.

\section{System Overview}

A polygonal heart model was acquired from the New York University's School of Medicine, which was designed by consultant cardiologists and graphical designers for the purpose of interactive teaching tools and VR applications. There were a few changes needed to make the model suitable for our system. These changes were mainly added in an earlier version of our software [8]. Firstly, key electrical network anatomy was added i.e. The SA (sino-atrial) node, the AV (atrioventricular) node and the bundle-branches were added. Also, the septal region was widened to allow for the bundle-branches. The SA and AV nodes were added as polygonal spheres using a commercial editor. The bundle-branches were added as lines directly using our own software. The volume lattice has a resolution of $256 \times 256 \times 100$. This is obviously not at cellular level, but, is considered sufficient for the purposes of this animation. The initial ECG data was acquired by recording a 12 lead ECG of a staff member. No pathologies were present.

Our initial model used points to represent each cell within the volume [8]. However, at the drawing stage it was very obvious that this method was too slow for the system, therefore an alternative approach was needed. Texture-based volumization is becoming very popular recently because of its fast rendering advantages [20][4]. In our earlier work [11] we developed a method of taking slice data from the original model and altering each manually for cell assignment. Therefore, instead of having a volume of separate points we have 100 slices of $256 \times 256$ resolution. The slice data is attained using a very thin viewing field and an orthographic projection. The image information was outputted to .tga files. The myocardial cells were represented with an opaque value and the blank cells were represented as a fully transparent values. Figures 4, 5 and 6 show this process of slice acquisition.

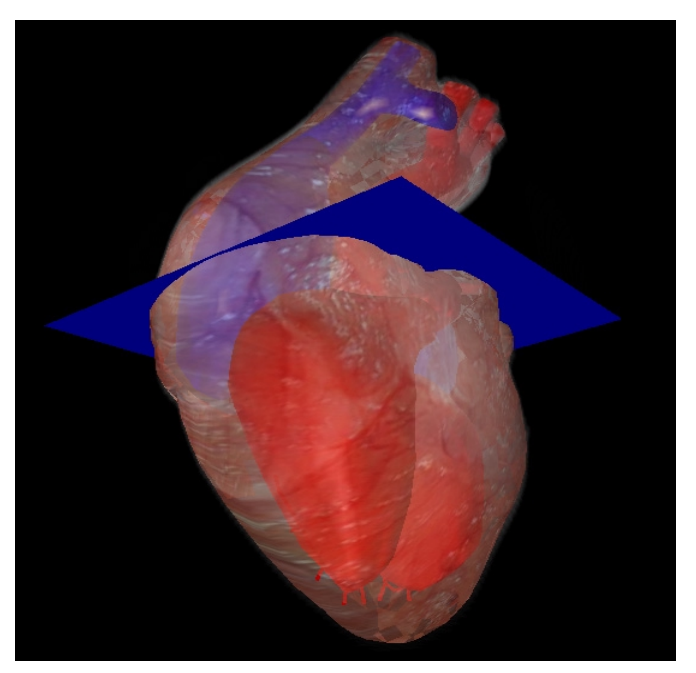

Figure 4. Location of viewing field. 


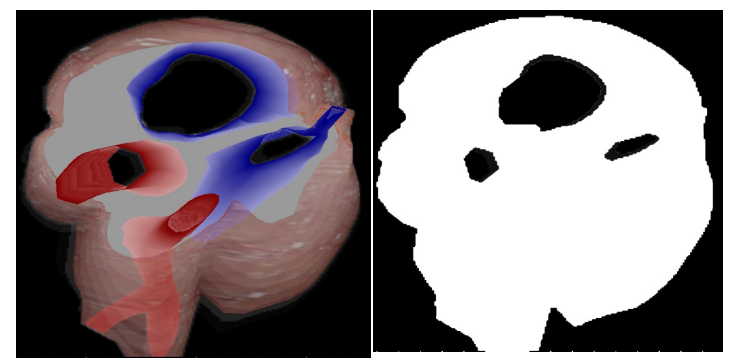

Figure 5 Slice as Figure 6. Typical slice viewed in openglwith dual colour window. representation.

Using the resulting images from this process the model's volume is displayed by reconstructing these slices along the same axis as the original viewing fields. This slicebased volume representation method is becoming widely accepted because of its speed advantages over other methods such as displaying cells as single points.

Each voxel within the volume has two main components, the cellular information and the pixel information. The pixel information tells the system about the color values and transparency and basic information about the different tissues. Whereas, the cellular data holds information about the specific cell types, timings and frame indices. The cell datastructure is currently open-ended to allow for more complex computations such as fully ionic modelling. Because this system is aimed at interactive tools the cell structure has to be simple. To simplify the electrical spread the system currently caters for a nearest-neighbour spread of the depolarising cells. There are five main types of cells at the moment: blank cells, atrial cells, ventricular cells, sino-atrial cells and atrio-ventricular cells. Bundle-branch injection cells will be added to represent parts of the bundle branch. The system is being developed to automatically detect and represent myocardial infarctions, therefore, specific locations mainly in the ventricles will be assign extra cell-types. Fibre orientations are currently not considered, but we aim to expand the cellular computations in the near future.

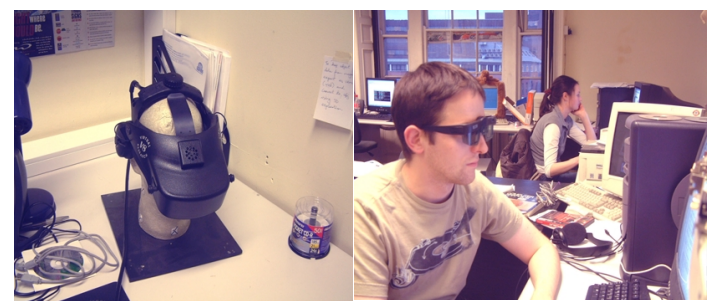

Figure 7(a). Standard (b). Polarized 3D dual LCD goggles Glasses

In order to give the user a greater sense of immersion we implemented a simple stereoscopic vision system. We chose not to use the dual screen goggles (figure 7a) because it was easier to implement the polarized 3D glasses (figure 7b). These glasses are very cost effective and do all the computations at driver level, therefore are very easy to implement. The glasses are synchronized with the CRT monitor to act as a shutter system. A different image is interleaved every second frame and shown to alternate eyes [18]. The software had to be converted to a GLUT (opengl windowing library) program instead of FLTK (graphical user inferface library that caters for opengl rendering). FLTK did not support stereoscopic vision.

\section{Animation and Synchronization}

A key feature with our system is that the representation of the electrical excitation of cells on the volumetric model runs in synchrony with the featured ECG. A set of pre-defined ECG markers is the basis of the animation. These markers may be defined either manually or automatically. The automatic wave classifier uses the So and Chan QRS detection algorithm [14]. Tan et al [15] found that the So and Chan QRS detection algorithm had a greater sensitivity than that of Pan and Tompkins [9]. So and Chan's method was also aimed at ambulatory ECG monitors for real-time detection. This quality is in keeping with our system's realtime specifications. Once the QRS marker is allocated we traverse forwards and backwards to find the individual $\mathrm{Q}, \mathrm{R}$ and $\mathrm{S}$ waveforms. Then, using the same thresholding technique of So and Chan, the $\mathrm{P}$ and $\mathrm{T}$ waveforms are 
found. There are other markers of key importance to the animation. These markers locate the end and start of the $\mathrm{P}$ and $\mathrm{T}$ waveforms $\left(\mathrm{P}_{\text {in }}, \mathrm{P}_{\text {out }}, \mathrm{T}_{\text {in }}\right.$ and $\left.\mathrm{T}_{\text {out }}\right)$. We defined the $\mathrm{P}_{\text {in }} / \mathrm{P}_{\text {out }}$ markers as the $\mathrm{P}$ markers $+/-\mathrm{a}$ difference of $\mathrm{Q}-\mathrm{P} / 4$ and the $\mathrm{T}_{\text {in }} / \mathrm{T}_{\text {out }}$ markers using the same difference but divided by 2 instead. These are approximations but any disadvantage is negligible. Figure 8 displays the ECG window of a normal ECG with defined markers.

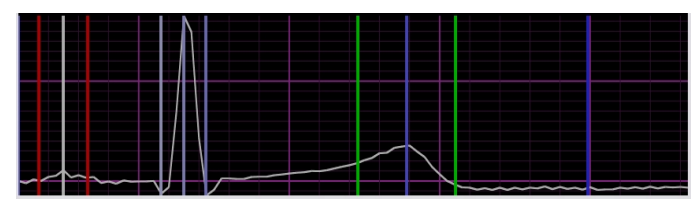

Figure 8. ECG window with markers

The electrical cycle begins with the SA node, which is represented in the polygonal mesh as a sphere and also in the volume as a single voxel. The SA node sphere flashes along with the preliminary firing of the cells in the vicinity of the SA node. With every iteration each cell's status is identified. If a certain cell is depolarized then its neighbouring cells are depolarized, thus, causing a spread of the electrical excitation. There is always a shape for a nearest-neighbour algorithm. We found that the simple 3D cross shape, as displayed in Figure 9, was sufficient.

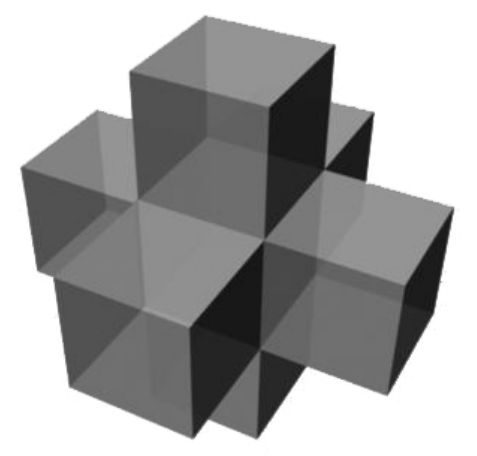

Figure 9. Nearest neighbour progression shape.

There are many conditions in the decision for electrical propagation. Apart from the depolarized/repolarized condition, the celltype is another large consideration. For example, when the progressing ECG marker is within the $P$ wave the atrial cells should be the only cells being activated. For this reason, it was necessary to categorize the volume into cell-types. This was done by differentiating each cell-type with a different color. We did this by manually editing each slice with a commercial editor. Currently we have 5 main cell-types which include blank, atrial, ventricular, sino-atrial and atrio-ventricular cells. Figure 10 shows an example of this with extra cell-types for a future diagnostic version of this software. Red represents atrial cells and blue represents ventricular cells. The other colors are representative of common myocardial infarction sites in preparation for our future diagnostic tool.

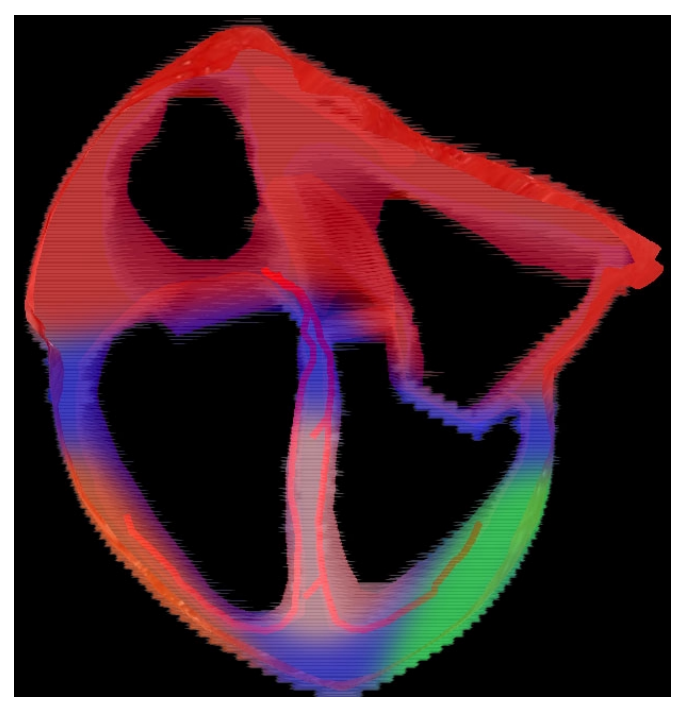

Figure 10. Cellular Classification - Frontal Cross-sectional view

The bundle-branches are displayed as a series of lines with insertion points within the septum and on the inferior borders of the ventricles.

There have been many different generations of this animation system. A previous version [11] used a pre-computed database of circles to display the isotropic spread on each slice. Although this ran quickly, it had several disadvantages regarding anatomical phenomena. This cellular spread ignored anatomical structures and spread right through the whole volume. The current spread, however, only spreads where there are other cells. 


\section{Results}

The ECG recognition and interpretation needs of our system benefits from the extra scope made available by using the slice-based technique. Another advantage of this method is aesthetics. This style of volume representation seems more uniform and generally looks better than its counterparts. Graphics hardware may be exploited to alter textures. This is yet another advantage of using this technique. Our earlier systems [8][11] depended on either 20 minutes of precomputations and displayed at a rate as slow as $2 \mathrm{fps}$ (frames per second) or ignored anatomical boundaries. The newer slice-based system displays a larger volume at between 10-20 fps with real-time computations. Figures 11 and 12 are graphical evidence of the aesthetic qualities of these two volumetric methods.

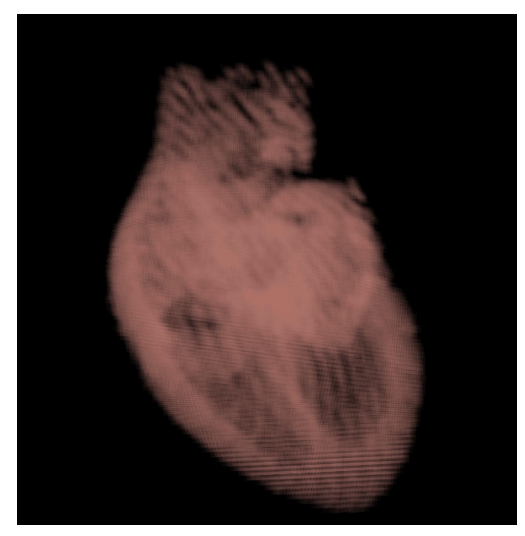

Figure 11. Voxel per group of cells

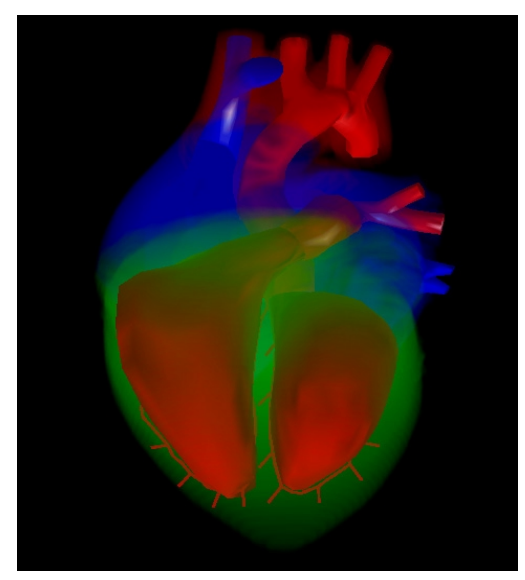

Figure 12. Textured slices
Speed optimization is an important issue with this software because of the large amount of processing required by volumetric animation. The importance of speed optimization is reinforced by the need for real-time interactivity within the system. To provide for this a frame indexing system was implemented to record the frame number where each cell is depolarized and repolarized. This information is collected during one iteration of the simulation and then reorganised into a speed friendly structure and outputted to a file for every other time the same ECG file is used. This frame indexing system has increased the frame rate from approximately $1 \mathrm{fps}$ to $20 \mathrm{fps}$. One of the major advantages of this method is that the cell structure can become more complex without affecting the animation speed, just the initial iteration.

The cellular progression algorithm is more accurate than previously, yet using the frame indexing technique, running speed is not compromised. In fact, the running speed has improved drastically. The ECG synchronization system is effective in defining markers with which to determine the exact points within the animation. Figure 13 demonstrates the electrical progression of a normal non-pathological ECG within our system. 


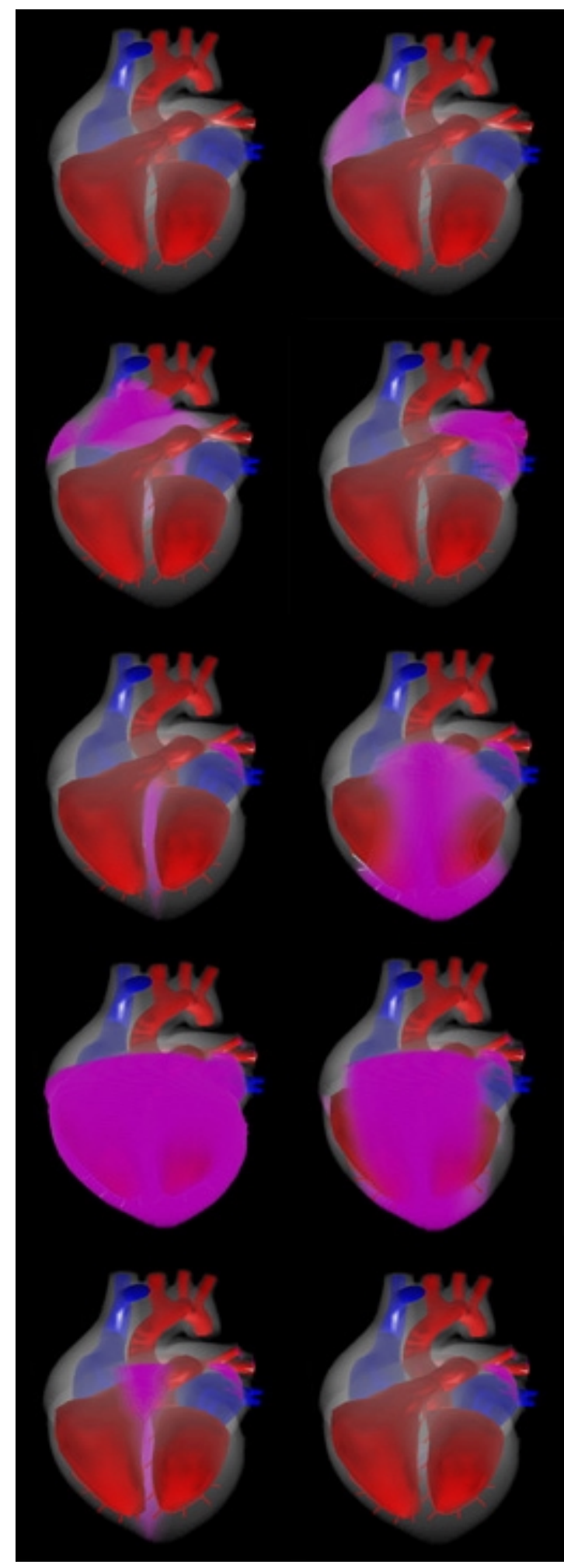

Figure 13. Animation example strip

\section{Conclusions and Future Work}

We have described a method for representing the electrical cycle of the heart with real-time, interactive, volumetric animation. This animation is synchronized automatically or manually with inputted ECG data. This animation system is part of a project entitled "A Virtual Reality Toolkit for the Monitoring of Myocardial Infarctions". We aim to create a diagnostic and treatment tool for cardiology practitioners in the field of myocardial infarction. We are working with cardiologists in a leading cardiology unit to devise a tool that is clinically relevant. In the future we aim to implement extra VR immersion technologies such as a haptic feedback arm to act as a virtual scalpel. Graphics hardware will also be exploited to carry some of the workload.

\section{References}

1. Dobson H.D., Pearl R.K., Orsay C.P., Rasmussen M., Evenhouse R., Ai Z., Blew G., Dech F., Edison M.I., Silverstein J.C, Abcarian H. (2003) Virtual Reality: New Method of Teaching Anorectal and Pelvic Floor Anatomy. Diseases of the Colon and Rectum. 46(3):349-352

2. Foley J B, Barry M, Crean P, Curtin R, Murphy RT, Pate GE, Talbot A, Walsh MJ and Ward D. (2002) Audit of Acute Myocardial Infarctions at St. James's Hospital, Dublin, From 1996 to 1999. Irish Medical Journal. 95(9):274-276

3. Kadambe S., Murray R., Boudreaux-Bartels G.F. (1999) Wavelet transform-based QRS complex detector. IEEE Transactions on Biomedical Engineering. 46:838-848

4. Kahler, R., Simon, M., Hege, H.C. (2003) Interactive Volume Rendering of Large Sparse Data Sets Using Adaptive Mesh Refinement Hierarchies. IEEE Transactions on Visualization and Computer Graphics, 9(3):341-351

5. Kennedy C. W., Hu T., Desai J. P., Wechsler A.S., Kresh J.Y. (2002) A Novel Approach to Robotic Cardiac Surgery Using Haptics and Vision. Cardiovascular Engineering: An International Journal. 2(1):16-22

6. Köhler B.U., Hennig C., Orglmeister R. (2002) The principles of QRS detection. IEEE 
Engineering in Medicine and Biology. January/February: 42-57

7. Kohl P, Noble D, Winslow R.L, Hunter P. (2001) The making of the virtual heart. In: Thompson, J.M.T. (ed.) Visions of the future: chemistry and life science. Cambridge: Cambridge University Press: 127-149.

8. Mooney, R, O'Sullivan, C. Ryan, J.T. and Bell, C. (2003) The Construction of a Volumetric Cardiac Model for Real-time ECG Simulation. Poster proceedings of the 11th International Conference in Central Europe on Computer Graphics, Visualization and Interactive Digital Media, pp 97-100

9. Pan J, Tompkins W. J. (1985) A real-time QRS detection algorithm. IEEE Trans. Biomed. Eng., BME-32 (3):230-236

10. Poli R., Cagnoni S., Valli G. (1995) Genetic design of optimum linear and non-linear QRS detectors. IEEE Transactions on Biomedical Engineering. 42:1137-1141

11. Ryan J, O’Sullivan C, Bell C, Mooney R (2004) A Virtual Reality Electrocardiography Teaching Tool. Proceedings $-2^{\text {nd }}$ International Conference, Biomedical Engineering. p250-253

12. Sara K. (1999) Thrombolytic agents in district hospitals. Eastern Mediterranean Health Journal. 5(1):182-185

13. Sermesant, M., Faris, O., Evans F., McVeigh E., Coudiére, Y., Delingette, H., Ayache, N. (2003) Preliminary validation using in vivo measures of a macroscopic electrical model of the heart. International Symposium on Surgery Simulation and Soft Tissue Modelling Lecture Notes. 2673:230-243

14. So, H.H., Chan, K.L. (1997) Development of QRS Detection Method for Real-Time Ambulatory Cardiac Monitor. Proceedings $-19^{\text {th }}$ International Conference - IEEE/EMBS: 289-292

15. Tan K F, Chan K L, and Choi K (2000) "Detection of the QRS complex, $\mathrm{P}$ wave and $\mathrm{T}$ wave in electrocardiogram," Proceedings of International Conference on Advances in Medical Signal and Information Processing, Bristol, UK, pp. $41-47$

16. Tatara E. and Cinar A. (2002) Interpreting ECG Data by Integrating Statistical and Artificial
Intelligence Tools. IEEE Engineering in Medicine and Biology. January/February: 42-57

17. Trahanias P., Skordalakis E. (1990) Syntactic Pattern Recognition of the ECG. IEEE Transactions on Pattern Analysis and Machine Intelligence. 12(7):648-657

18. Website: Cambridge Research Systems, Stereopsis: http://www.crsltd.com/apps/stereo/ Accessed: 2PM, 2/12/03

19. Website: CESLab cardiac electrophysiological simulation for Apple Mac's, http://www.cesinst.com Accessed: 11AM, 20/2/04

20. Weiskopf, D., Engel, K., Ertl, T., (2003) Interactive Clipping Techniques for TextureBased Volume Visualisation and Volume Shading. IEEE Transactions on Visualization and Computer Graphics, 9(3):298-312

21. Xue Q., Hu Y.H., Tompkins W.J. (1992) Neural Network-based adaptive matched filtering for QRS detection.IEEE Transactions on Biomedical Engineering. 39:317-329

\section{Acknowledgements}

This project is funded by Enterprise Ireland under the Basic Research Grant scheme. We gratefully acknowledge the assistance of Dr. Peter Crean.

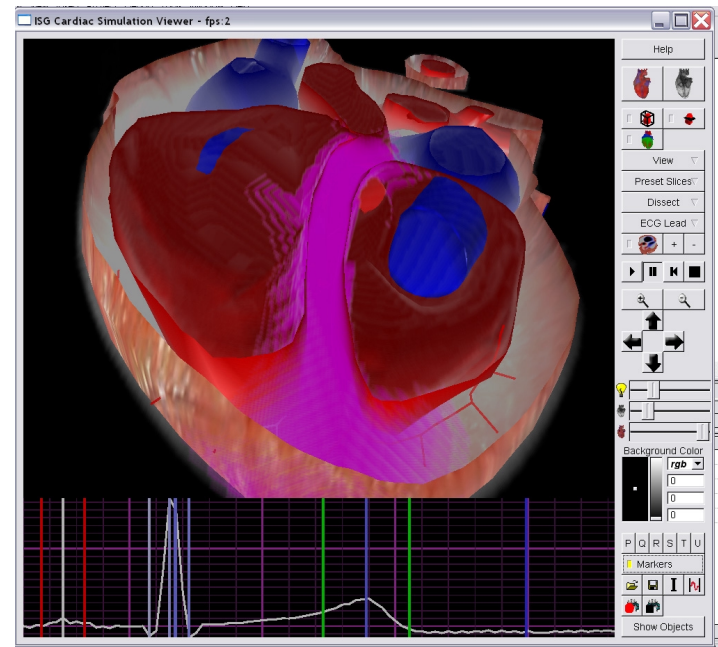

Figure 14. Screenshot of system, see also accompanying video. 